NOTA CIENTÍFICA

\title{
Nematofauna de tres especies de lagartijas (Sauria: Tropiduridae y Gekkonidae) de la Reserva Nacional de Paracas, Ica, Perú
}

\section{Nematofauna of three species of lizards (Sauria: Tropiduridae and Gekkonidae) from National Reserve Paracas, Ica, Peru}

\begin{abstract}
${ }^{1}$ Museo de Historia Natural. Universidad Nacional Mayor de San Marcos. Lima. Perú.

2 Laboratorio de Estudios de Biodiversidad. Departamento Académico de Ciencias Biológicas y Fisiológicas. Facultad de Ciencias y Filosofía. Universidad Peruana Cayetano Heredia. Lima. Perú

3 Departamento de Ecología. Instituto de Biologia Roberto Alcântara Gomes. Universidade do Estado do Rio de Janeiro. Rio de Janeiro. Brasil.

E-mail, José Pérez Z.:

perezz_jm@yahoo.com.br
\end{abstract}

Presentado: $\quad 06 / 10 / 2006$ Aceptado: $\quad 12 / 01 / 2007$

\author{
José Pérez Z., ${ }^{1,23}$, Katya Balta ${ }^{1,2}$, Patricia Salizar¹ y Lidia Sánchez ${ }^{1}$
}

\section{Resumen}

Los nemátodos Thubunaea iguanae y Spauligodon viracochai son reportados para tres especies de lagartijas en la Reserva Nacional de Paracas, Ica, Perú. El primer nematodo fue registrado para Microlophus peruvianus y Microlophus thoracicus thoracicus, mientras que el segundo fue registrado para Phyllodactylus angustidigitus. Estos reportes representan dos nuevos hospederos y una nueva localidad para T. iguanae, nematodo reportado por primera vez para Sudamérica, además del registro de un nuevo hospedero para S. viracochai.

Palabras clave: Thubunaea iguanae, Microlophus peruvianus, Microlophus thoracicus thoracicus, Phyllodactylus angustidigitus, Spauligodon viracochai, Reserva Nacional de Paracas, Ica, Perú.

\section{Abstract}

The nematode Thubunaea iguanae and Spauligodon viracochai are reported for three species of lizards from Paracas National Reserve, Ica, Peru. The first nematode was found on Microlophus peruvianus and Microlophus thoracicus thoracicus while the second one was found on Phyllodactylus angustidigitus. Reports are two new host records and a new locality record for T. iguanae, and a first record for South America, and a new host for S. viracochai.

Keywords: Thubunaea iguanae, Microlophus peruvianus, Microlophus thoracicus thoracicus, Phyllodactylus angustidigitus, Spauligodon viracochai, National Reserve Paracas, Ica, Peru.
Existe poca información sobre los parásitos de los reptiles del Desierto Costero Peruano. Sólo se conocen cinco reportes para localidades en el norte (Baylis, 1926; Freitas e Ibáñez, 1963; Rêgo e Ibañez, 1965; Freitas et al., 1968a; Freitas et al., 1968b) y dos para el sur de esta ecoregión (Calisaya y Córdova, 1997; Salas y Campos, 1974). A la fecha no se conocen evaluaciones realizadas sobre los parásitos de reptiles para localidades en el Desierto Costero Peruano central (Ancash, Lima, Ica).

Las especies reportadas en el presente trabajo fueron encontradas en tres especies de saurios procedentes de la Reserva Nacional de Paracas y colectados durante una evaluación ecológica de reptiles en esta reserva (Pérez y Balta, 2007).

Los estómagos de las lagartijas fueron examinados con ayuda del microscopio estereoscópico. Los nemátodos hallados fueron fijados en formol 10\%, preservados en alcohol 70\%, y clarificados en alcohol-fenol. Todas las medidas de los helmintos se realizaron con un ocular micrométrico. Los helmintos se depositaron en la Colección Helmintológica y de Invertebrados Relacionados del Departamento de Protozoología, Helmintología e Invertebrados Afines del Museo de Historia Natural de la Universidad Nacional Mayor de San Marcos. Las lagartijas se depositaron en la Colección del Departamento de Herpetología del mismo museo (M. peruvianus: MUSM 24569, 24570, 24571; M. $t$. thoracicus: MUSM 24572, 24573, 24574 y P. angustidigitus: MUSM 24575).

Se registró a Thubunaea iguanae Telford (Nematoda: Spiruridea: Physalopteridae) en la lagartija de playas Microlophus peruvianus Lesson y en la lagartija de gramadales $M$. thoracicus thoracicus Tschudi, ambos saurios pertenecientes a la familia Tropiduridae. Adicionalmente, se registró a Spauligodon viracochai Freitas e Ibañez (Nematoda: Pharyngodonidae) en el geko de Paracas Phyllodactylus angustidigitus Dixon y Huey de la familia Gekkonidae. Se revisaron diez individuos de M. peruvianus, registrándose nematodos en tres especimenes (Prevalencia: $30 \%$ ). En el caso de M. t. thoracicus se revisaron seis individuos, registrándose nematodos en tres especimenes (Prevalencia: 50\%). Se revisaron siete individuos de $P$. angustidigitus, registrándose nematodos en sólo un individuo (Prevalencia: 14\%).

Se colectaron un total de 110 nematodos de la especie Thubunaea iguanae de los estómagos de $M$. peruvianus (38 nematodos) y $M$. t. thoracicus (72 nematodos). Los nematodos coincidieron con las características descritas por Telford (1965), como la posición de la vulva, número y distribución de las papilas caudales, longitud de las espículas y medida de los huevos. También se colectaron siete nematodos hembras en estómago de P. angustidigitus, cuyas características coinciden con Spauligodon viracochai (Freitas e Ibañez, 1968).

\section{Thubunaea iguanae (Telford, 1965)}

Descripción: La longitud total del cuerpo de las hembras es de $17,63 \mathrm{~mm}$, con un ancho de $0,36 \mathrm{~mm}$, faringe de 0,04 de longitud, seguida del esófago de 1,40 de longitud. El anillo nervioso está ubicado a $0,17 \mathrm{~mm}$ del extremo anterior. La longitud total del cuerpo de los machos de 9,20 mm, con un ancho de $0,26 \mathrm{~mm}$, faringe de $0,03 \mathrm{~mm}$ de longitud, y seguida del esófago de $1,14 \mathrm{~mm}$ de longitud. El anillo nervioso está ubicado a 0,15 $\mathrm{mm}$ del extremo anterior.

Las hembras presentan la vulva ubicada a $1,90 \mathrm{~mm}$ del extremo anterior, mientras que el ano y el recto están a 0,20 y 0,03 del extremo posterior. Los machos presentan 9 pares de papilas pedunculadas y 6 pares sésiles, distribuidas como sigue: 5 pares 
pedúnculas y 1 par sésil preanal, 1 par pedunculado y 2 pares sésiles adanales, 3 pares pedunculadas y 3 pares sésiles postanales. Presenta un par de espículas desiguales de 0,15 y $0,13 \mathrm{~mm}$.

Hospedero: Microlophus peruvianus (Lesson, 1826)

Número de Hospederos examinados: 10

Prevalencia: 30\% (3 individuos)

Número de Helmintos colectados: 38 individuos (5 machos y 33 hembras).

Localidad: Reserva Nacional de Paracas, provincia de Pisco, departamento de Ica, Perú.

Localización: Estómago

$\mathrm{N}^{\circ}$ Col. Helm. MUSM 2187, 1 vial

Hospedero: Microlophus thoracicus thoracicus (Tschudi, 1845)

Número de Hospederos examinados: 6

Prevalencia: $50 \%$ (3 individuos)

Número de Helmintos colectados: 72 individuos $(5$ machos y 67 hembras).

Localidad: Reserva Nacional de Paracas, Pisco, Ica, Perú.

Localización: Estómago

$\mathrm{N}^{\circ}$ Col. Helm. MUSM 2188, 1 vial

\section{Spauligodon viracochai (Freitas \& Ibáñez, 1968)}

Descripción: La longitud total del cuerpo de las hembras es $3,16 \mathrm{~mm}$ con un ancho de 0,33 $\mathrm{mm}$. Esófago con 0,38 $\mathrm{mm}$ de longitud. El anillo nervioso está ubicado a $0,08 \mathrm{~mm}$ del extremo anterior. El poro excretor y la vulva están ubicados a 0,36 y 0,46 $\mathrm{mm}$ respectivamente.

Hospedero: Phyllodactylus angustidigitus (Dixon \& Huey, 1970)

Número de Hospederos examinados: 7

Prevalencia: 14\% (1 individuo)

Número de Helmintos: 7 hembras

Localidad: Reserva Nacional de Paracas, Pisco, Ica, Perú.

Localización: Estómago

$\mathrm{N}^{\circ}$ Col. Helm. MUSM 2300, 1 vial

Thubunaea iguanae difiere de T. intestinales y T. parkeri debido a que este último, descrito por Baylis (1926) para Microlophus occipitalis y Dicrodon heterolepis (sin. Dicrodon calliscelis), presenta 8 a 10 pares de papilas, de las cuales 5 pares son preanales y pocas post anales, además no presenta espícula. Mientras que T. intestinales, reportada por Bursey \& Golberg (1991) para Sceloporus jarrovii, se caracteriza por presentar 15 pares de papilas: 4 pares pedunculadas preanales y 4 pares pedunculadas postanales, 3 pares sesiles preanales y 4 pares sesiles postanales; sus espículas son desiguales y miden 0,055 y $0,038 \mathrm{~mm}$ de longitud. Thubunaea ctenosauri registrada por Moravec et al. (1997) difiere de T. iguanae por presentar 16 a 17 pares de papilas distribuidas de la siguiente forma: 8 pares preanales, 2 pares adanales y 6 ó 7 pares postanales, además presenta espículas iguales ligeramente esclerotizadas de 0,066 $\mathrm{mm}$ de longitud.

El presente registro representa nuevos hospederos y nueva localidad para Thubunaea iguanae, reportado por primera vez por Telford (1965) para varias especies de lagartijas de Norte América. Esta especie no había sido registrada en ninguna lagartija del Desierto Costero Peruano, siendo también el primer registro para Sudamérica. Para $M$. peruvianus se ha reportado a Parapharyngodon yurensis, P. moqueguensis, P. arequipensis (Cali- saya y Córdova, 1997), y Thelandros bulbosus (Salas y Campos, 1974). Por lo tanto, con nuestro reporte se incrementarían a cinco el número de especies de nemátodos registrados en Perú para M. peruvianus. Sin embargo, cabe destacar que la distribución conocida de $M$. peruvianus se extiende a lo largo del Desierto Costero Peruano desde Tumbes hasta Arequipa (Dixon y Wrigth, 1975; Carrillo e Icochea, 1995), por lo tanto deben confirmarse las identificaciones de las lagartijas hospederas de los parásitos registrados para el departamento de Moquegua, específicamente P. moqueguensis y P. arequipensis, reportados por Calisaya y Córdova (1997). Thubunaea iguanae es el primer parásito reportado para M. t. thoracicus.

Spauligodon viracochai registrado por primera vez por Freitas e Ibáñez (1968a) para Phyllodactylus gerrhopygus, encuentra un nuevo hospedero en Phyllodactylus angustidigitus. Si bien para este reporte sólo se contó con nemátodes hembras, todas las características coinciden con las proporcionadas por Freitas e Ibáñez (1968a) para esta especie, como la posición de la vulva y el poro excretor.

\section{Agradecimientos}

Agradecemos al Dr. Charles Bursey del Departamento de Biología de la Universidad Estatal de Pensilvania U.S.A. y Dr. Guillermo Salgado Maldonado del Instituto de Biología de la Universidad Autónoma de México, por facilitarnos referencias bibliográficas y sugerencias para el trabajo. A un revisor anónimo por las sugerencias a esta nota científica. A Eliana Quispitúpac, Emilio Fuentes y Alberto Guanilo por la ayuda en el trabajo de campo en la colecta de las lagartijas. Al personal de la Reserva Nacional de Paracas por el apoyo logístico, en especial a Luis Paz Soldán, Víctor Quispe y Juan Heaton. Al Grupo de Estudios Ambientales del Perú (GEA-PERU) y World Wildlife Fund - Oficina Programa Perú (WWF-OPP) por el financiamiento de la fase de campo de este proyecto.

\section{Literatura citada}

Baylis, H. 1926. On a new Species of the Nematodo Genus Thubunaea Ann \& Mag. N. Hist. 9 (18): 361-364.

Bursey, C. \& S. Goldberg. 1991. Thubunaea intestinalis n. sp. (Nematoda: Spiruroidea) from Yarrow's Spiny Lizards, Sceloporus jarrovii (Iguanidae), from Arizona, U.S.A. Trans Am. Microsc. Soc. 110(3):269-278.

Calisaya, J. \& E. Córdova. 1997. Tres Nuevas Especies de Parapharyngodon (Nematoda, Oxyuroidea) parásitas de Tropidurus peruvianus. REBIOL. 17 (1 y 2): 45-55.

Carrillo, N. \& J. Icochea. 1995. Lista Taxonómica Preliminar de los Reptiles vivientes del Perú. Publ. Mus. Hist. Nat. UNMSM (A) 49: 1-27.

Dixon, J \& J. Wright. 1975. A review of the lizards of the iguanid genus Tropidurus in Peru. Contribution in Science. The Natural History Museum of Los Angeles. 40 p.

Freitas, J. F. T. \& N. Ibáñez, 1963. Fauna Helmintológica del Perú - Notas sobre "Pharyngodonidae" Travassos, 1920 y descripción de Pharyngodon micrurus sp. $n$. Separata de "Revista Universitaria" $N^{\circ} 23$ y 24.

Freitas, J. F. T.; J. J. Vicente \& N. Ibáñez. 1968a. Fauna helmintológica do Peru: Novo nematódeo do gênero "Parathelandros" Baylis, 1930 (Nematoda, Oxyuroidea). Atas Soc. Bio. Rio de Janeiro. 12 (1): 33-35.

Freitas, J. F. T.; J. J. Vicente \& N. Ibáñez. 1968b. Fauna Helmintológica del Perú: Parathelandros capacyupanquii sp. n., parásito de Dicrodon holmbergi Schmidt, 1957 (Nematoda, Oxyuroidea). Atas Soc. Bio. Rio de Janeiro. 11 (6): 217-219. 
Moravec, F; G. Salgado-Maldonado \& E. Mayen-Peña. 1997. Thubunaea ctenosauri sp. n. (Nematoda: Physalopteridae) from the Iguanid Lizard Ctenosaura pectinata and Other Lizard. J. Helminthol. Soc. Wash. 64(2): 240-247.

Pérez Z., J. y K. Balta. 2007. Ecología de la comunidad de saurios diurnos de la Reserva Nacional de Paracas, Ica, Perú. Revista Peruana de Biología. 13(3): 169-176.

Rêgo A. A. \& N. Ibáñez. 1965. Duas novas espécies de Oochoristica, parásitas de lagartixas do Peru (Cestoda, Anoplocephalidae). Mem Inst. Oswaldo Cruz 63: 67-73.

Salas \& Campos, 1974. Descripción del Oxyuroide Thelandros bulbosus encontrados en Lagartos de la Región de Arequipa. Libro de Resúmenes del IV Congreso Nacional de Biología. pp. 125.

Telford, S. 1965. A New Species of Thubunaea (Nematoda: Spiruroidea) from California Lizards. Japan J. Exp. Med. 35 (2): 111-114. 
